\title{
Valores normativos para el programa de análisis acústico del habla Motor Speech Profile en hablantes de español de Chile
}

\author{
Normative Values for the Acoustic Speech \\ Software Motor Speech Profile in Chilean spanish \\ Speakers
}

Hernán Pérez M.

Doctor en Lingüística

Director del Laboratorio de Fonética Universidad de Concepción, Chile

Rocío Fernández 0. Fonoaudióloga Magíster en Lingüística Aplicada Universidad de Concepción, Chile

Carlos Oliva F. Ingeniero Electrónico

Contacto con el autor:

Rocío Fernández $\mathrm{O}$.

Victoria 1472, casa 10.

Santiago - Chile

Tel: (56 9) 56597918

Correo-e: rociofernandezo@hotmail.cl

Recibido: 22/01/2015

Aceptado: 21/09/2015

\section{RESUMEN}

El objetivo de este artículo es exponer los resultados de un estudio normativo del rendimiento articulatorio de individuos chilenos normales, medido con los cinco parámetros que entrega el protocolo de rendimiento diadococinético del programa Motor Speech Profile de KayPentax. La intención es proporcionar a la comunidad fonoaudiológica valores de referencia de una muestra de hablantes del país para que puedan ser utilizados en la aplicación clínica y académica de este instrumento. Mediante el procedimiento de muestra espejo, se replica el estudio con el que se obtuvieron los valores de referencia originales del programa en una muestra de hablantes de inglés norteamericano. Además, se comparan ambos estudios con el propósito de determinar las diferencias y similitudes en el rendimiento de cada uno de los parámetros. Se concluye que tanto el DDKcvp como el DDKcvi manifiestan un comportamiento similar en ambos grupos y que el resto de los parámetros presenta ciertas diferencias. No obstante, en todos los parámetros es posible establecer un rango de variación que permite definir el comportamiento normal.

Palabras clave: rendimiento diadococinético, Motor Speech Profile, rango de variación.

\section{ABSTRACT}

The present study aim to provide normative data of articulatory performance in normal Chilean individuals. The normative values obtained can serve as reference values from Chilean speakers which the speech-language pathology community can use in clinical and academic practice. The five parameters defined in the diadochokinetic protocol in the Motor Speech Profile of the KayPentax program were used. The method employed in the study which obtained the reference values from America English speakers was also adopted in this study. Furthermore, a comparison between the English and Spanish studies was carried out.

DDKcvp and DDKcvi showed similar behavior in both groups. The other parameters presented some differences. However, a normal range for all parameters was possible to obtain.

Keywords: diadochokinetic protocol, Motor Speech Profile, variation range. 


\section{Introducción}

En las últimas décadas, ha aumentado entre los especialistas el interés por el análisis cuantitativo instrumental en la evaluación clínica de la función motora. Esto ocurre porque poco a poco ha ido tomando mayor fuerza la idea de que este tipo de análisis ofrece potencialmente una mayor economía de tiempo, mayor fiabilidad e información más detallada de la que se puede lograr con los métodos tradicionales que se basan, fundamentalmente, en la apreciación subjetiva del terapeuta. En efecto, de forma tradicional la evaluación y diagnóstico de los trastornos del habla se realizan en principio solamente escuchando el habla del individuo, aunque con la ayuda de protocolos y pautas de cotejo ya establecidos, usados, validados, aceptados y conocidos ampliamente por la gran mayoría de los terapeutas.

Sin duda, es necesario y pertinente que los profesionales del habla y del lenguaje determinen el diagnóstico y avances de una terapia por medio de su propia percepción y experiencia. No obstante, debido al desarrollo que ha alcanzado la tecnología actualmente, se hace recomendable también que, además, respalden sus juicios, opiniones y resultados por medio de métodos de análisis objetivos y fiables. Esto, no solo porque de esta manera se le da más validez a una evaluación y a los resultados de la terapia, sino que también porque tener datos cuantitativos de análisis acústico permite realizar una fácil comparación con resultados obtenidos anteriormente, o con los que se obtendrán más adelante.

La accesibilidad y el bajo costo han llevado a los métodos perceptivos a ser la herramienta fundamental para realizar diagnósticos diferenciales, establecer grados de severidad (por medio de escalas de inteligibilidad, mediciones de tasa del habla y de precisión articulatoria) y elaborar planes de rehabilitación. Estos métodos se basan en la observación auditiva y visual de la señal emitida por el hablante. Así, la eficacia del método depende, en gran medida, del conocimiento, la experiencia y habilidad del clínico, así como también de los factores aportados por la situación y por el paciente.

Los métodos de análisis acústico, al igual que los métodos subjetivos, toman como objeto de su análisis la señal emitida. No obstante, permiten cuantificar varios rasgos y características de la señal acústica, mediante lo cual se pueden obtener diversas medidas objetivas para establecer una línea de base en la evaluación y determinar luego los avances de la rehabilitación del habla.

Para realizar un análisis acústico es necesario disponer de elementos mínimos para extraer una muestra del habla, los que generalmente están dados por el software en cuestión. Para que todos los componentes tecnológicos trabajen de forma armónica debe existir un equilibrio en sus características de requerimiento. Es importante notar que es preferible siempre trabajar con elementos que estén por sobre la norma de requerimiento mínimo, ya que así es posible garantizar que los datos obtenidos son reales y que no existen factores externos que pudieran alterarlos.

\section{El índice de diadococinesia}

El rendimiento articulatorio del habla de un individuo se evalúa mediante técnicas subjetivas y objetivas. Los procedimientos subjetivos se basan en 
observaciones de las tareas articulatorias realizadas por la persona evaluada y sustentadas en la percepción del evaluador. Dentro de estas técnicas se encuentran los test de articulación en habla espontánea, repetición de palabras y la diadococinesia articulatoria perceptual (Queiroz, 2002).

A partir de la década de los cincuenta, surgen parámetros que se pueden medir de forma instrumental, los cuales permiten eventualmente establecer índices precisos de normalidad o deterioro. Sin duda, estos índices contribuyen a complementar la percepción del evaluador. Uno de ellos corresponde a la tasa de diadococinesia o tasa máxima de repetición, que ha sido considerada como uno de los procedimientos más aceptados para evaluar el rendimiento articulatorio del habla (Williams y Stackhouse, 2000). La tasa de diadococinesia corresponde a una medición cuantitativa de la capacidad de las personas para ejecutar voluntaria, alternada y rápidamente movimientos sucesivos y opuestos de los órganos articulatorios. Además, no solo se evalúa la capacidad para producir rápidamente un estímulo, sino que también se examina la regularidad de la producción, enfocándose en las variaciones presentadas y en el control de la intensidad (Lundy, Roy, Xue, Casiano y Jassir, 2004).

En Chile, Toledo et al. (2011) realizan un estudio normativo con el propósito de comparar el rendimiento en tareas de habla de adultos residentes en la Región Metropolitana según edad y género. Entre varios otros parámetros, miden el rendimiento diadococinético mediante el cálculo de la producción de sílabas por unidad de tiempo (en este caso, 5 segundos). Sus resultados indican que en promedio los sujetos normales producen aproximadamente 33 sílabas en 5 segundos, en tareas de diadococinesias con la sílaba "pa". Sus resultados señalan también que este parámetro es sensible a las diferencias de edad y género.

El índice de diadococinesia articulatoria es, por lo tanto, un correlato de la eficacia articulatoria e inteligibilidad del habla (Williams y Stackhouse, 2000). Este índice de evaluación de las habilidades motoras del habla es consistente en todas sus formas de aplicarse, esto es, en la articulación de unas 30 sílabas, serie de sílabas, vocal aislada o pares de vocales en forma rápida, con intensidad vocal constante y con un ritmo adecuado (Lundy et al., 2004).

\section{Motor Speech Profile (MSP)}

Dentro de los programas de análisis acústico que existen actualmente, se destaca el programa Motor Speech Profile (MSP), que forma parte del paquete de software Sona-Speech II v. 2.7 .0 (2004) de KayPentax. Este programa proporciona un análisis multidimensional de la conducta motora del habla con presentación gráfica y numérica de los resultados de análisis. Posee cinco protocolos de análisis del comportamiento del habla y seis en la opción avanzada:

- Rendimiento diadococinético

- Transición del segundo formante

- Temblor de la voz

- Estímulos de entonación

- Tasa de estándar silábico

- Tasa genérica silábica 
La tasa de diadococinesias y el coeficiente de variación del período están estrechamente asociados con la capacidad articulatoria, es por ello que este programa ha tomado un papel central en estudios actuales relacionados con patologías del habla, al incluir un análisis más detallado de sus procesos motores, especialmente del rendimiento diadococinético.

El protocolo de evaluación del rendimiento diadococinético consiste en solicitar al individuo que emita una secuencia consonante vocal ("pa") y que la repita de manera rápida y consistente. Esta emisión se graba y se procesa mediante el MSP, el cual entrega cinco parámetros numéricos que son los siguientes:

a) DDKavp /ms/ (índice de duración promedio de la sílaba). Este indicador mide y entrega el valor promedio de la duración de la sílaba, es decir, es el tiempo promedio, medido en milisegundos (ms), entre cada una de las unidades CV (esto es, cada vocalización “pa”). El DDKavp es inversamente proporcional al DDKavr.

b) DDKavr (promedio de sílabas por segundo o tasa de diadococinesias) / s/s /. Este es el promedio de la producción de sílabas en una unidad de tiempo durante la vocalización del individuo. Definido de manera más específica, es el número de combinaciones CV (es decir, “pa” vocalizaciones) por segundo. Este indicador está inversamente relacionado con el DDKavp.

c) DDKcvp (coeficiente de variación del período DDK o porcentaje de variabilidad en la producción de sílabas CV en un lapso de tiempo) /\%/. Este parámetro mide, en términos porcentuales, el grado de variación del promedio de sílabas por segundo. Si la vocalización $\mathrm{CV}$ se repite con poca variación en la unidad de tiempo, entonces este número es muy pequeño. Sin embargo, a veces puede variar la cantidad de sílabas producidas en cada unidad de tiempo durante la ventana de siete segundos de análisis, en ese caso, este valor aumenta.

d) DDKjit (perturbaciones del período DDK o porcentaje de variabilidad en la producción de sílabas CV, considerando ciclo a ciclo) /\%/. Este parámetro mide, en términos porcentuales, el grado de variación ciclo a ciclo en el período. Si la vocalización $\mathrm{CV}$ se repite con poca variación de la velocidad ciclo a ciclo (duración en milisegundos), entonces este valor es muy pequeño. Sin embargo, si la duración de cada sílaba es muy variable durante la ventana de siete segundos de análisis, este valor aumenta.

e) DDKcvi (coeficiente de variación de la intensidad máxima DDK) /\%/. Este parámetro mide, en términos porcentuales, el grado de variación de la intensidad en el pico de amplitud de cada vocalización CV. Si la vocalización $\mathrm{CV}$ se repite con poca variación en la intensidad, entonces este valor es bajo. Sin embargo, si un hablante varía la intensidad de DDK durante la ventana de siete segundos de análisis, este número aumenta. Este parámetro está evaluando la capacidad del sujeto para mantener una amplitud constante (es decir, la intensidad) durante la tarea de combinaciones de ciclos CV. 
Todos estos indicadores son expresados en el programa mediante gráficos como los que se ilustran en la Figura 1, en donde la línea verde oscura señala el valor medido al sujeto; la línea verde clara indica el valor promedio normativo dentro de los valores que trae consigo el software y el cuadrado amarillo indica el rango de dispersión permitido dentro de los valores normativos que vienen con el programa.

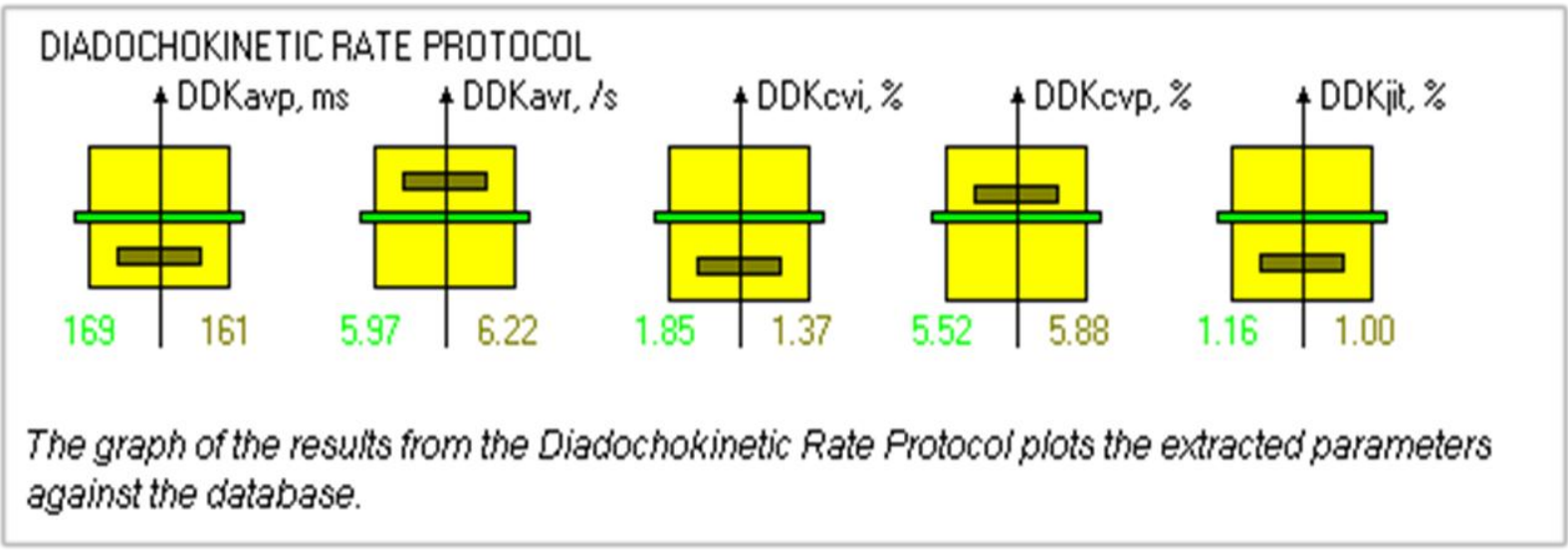

Figura 1. Gráficos de resultados del protocolo de diadococinesias en el programa MSP.

A pesar de que la investigación sobre algunos de estos parámetros está recién comenzando, todos parecen tener una conexión intuitiva con la evaluación de la ejecución motora del habla. Por ejemplo, la tasa de diadococinesias (DDKavr) se complementa con el cálculo de la periodicidad de la tasa (DDKcvp). Intuitivamente, esto se debe correlacionar con la capacidad del sujeto para producir rítmica y repetidamente la tarea articulatoria solicitada por el evaluador. Los estudios iniciales han demostrado que la periodicidad del DDK es un indicador sensible de algunos problemas motores del habla.

Al respecto, Meurer, Wender, Corleta y Capp (2004) realizan un estudio utilizando el programa MSP, en el cual comparan las características fonoarticulatorias de mujeres norteamericanas en edad reproductiva con mujeres en posmenopausia. Los resultados muestran que las mujeres en posmenopausia poseen un habla más lenta y tienen una menor estabilidad vocal.

Ese mismo año, en el Departamento de Otorrinolaringología de la Universidad de Miami, se realiza un estudio (Lundy et al., 2004) en el que se intenta diferenciar tres patologías que presentan dificultades del habla muy similares. Su propósito es determinar si las características vocales de origen neurológico podrían diferenciarse sobre la base de los parámetros acústicos y motores del habla. Para ello se analizó a tres grupos de sujetos (con disfonía espasmódica, esclerosis lateral amiotrófica y tremor vocal) con el software Motor Speech Profile. EI estudio concluye que cada grupo presenta características propias que lo diferencian de los demás.

Harel et al. (2004) realizan, mediante el análisis de diversos parámetros acústicos como el VOT, un estudio de seguimiento de pacientes con 
enfermedad de Parkinson. Sus resultados sugieren que los cambios tempranos del habla son signos detectables mediante ciertas medidas acústicas.

Por su parte, Brisso (2007) se interesa en el estudio de la tasa de diadococinesias en sujetos con fisura labiopalatina y compara los seis parámetros del índice de DDK entregados por el programa MSP en sujetos con fisura y sin fisura. Sus resultados no arrojan diferencias significativas, lo que indica que las diferencias anatómicas no son relevantes en este tipo de tareas, sin embargo, se apreció que en sujetos de menor edad con fisura existen mayores dificultades.

Vergara (2008) establece datos normativos de la tasa de diadococinesia medidos con el programa MSP en niños típicos hablantes del español (residentes en la ciudad de Talca, Chile) de entre 10 años y 13 años 11 meses.

Por otro lado, Padovani, Gielow y Behlau (2008) exponen un interesante estudio donde se utiliza el programa MSP para analizar el habla en sujetos normales brasileños. El objetivo de esta investigación fue comparar el desempeño diadococinético de 23 adultos jóvenes y 23 adultos mayores. Ambos grupos presentaron un DDK reducido a medida que la producción del habla se hacía más compleja y el grupo de adultos jóvenes presentó un DDK más adecuado en todas las tareas.

Entre los diferentes subtipos de disartria se encuentra la disartria atáxica que se ha definido especialmente por presentar una disdiadococinesia. Es por ello que Wang, Kent, Duffy y Thomas (2008) realizan un análisis de estos pacientes. En el estudio, participaron 21 individuos con disartria atáxica, a quienes se les aplicó el protocolo de diadococinesias (DDK); sin embargo, más de un tercio de las muestras fueron no ejecutables, debido a que este tipo de disartria se caracteriza, precisamente, por una marcada disdiadococinesia.

Entre las investigaciones actuales, Louzada, Beraldinelle, Berretin y Brasolotto (2011), especialistas del habla en la Universidade de São Paulo, han estudiado a personas con disfonía, intentando estrechar los límites entre el análisis del habla y de la voz. Se buscó comparar el rendimiento de DDK en mujeres con y sin disfonía. Los resultados no muestran variaciones significativas entre las poblaciones, sin embargo, el coeficiente de variación del período es más elevado en las mujeres disfónicas.

Por otra parte, Jeen, Kanaka, Sunila y Bellur (2014) han efectuado estudios normativos en la India. El objetivo principal de su trabajo fue establecer valores normativos para 18 parámetros del Motor Speech Profile en una parte de la población de este país, específicamente en los hablantes adultos de lengua kannada (o canarés), categorizados por edad y género. Los hallazgos de esta investigación sirven como referencia basada en la norma para el software de MSP en la población hablante de kannada, con edades comprendidas entre 20 y 60 años.

Considerando todos los antecedentes ya expuestos, se puede concluir que es necesario llevar adelante en Chile estudios que proporcionen valores de referencia para la aplicación y el uso del protocolo de diadococinesias del MSP, teniendo en cuenta además que este software se encuentra habilitado ya desde hace tiempo en varios centros clínicos 
públicos y privados y en un número considerable de instituciones de educación superior que forman fonoaudiólogos en el país.

Los resultados que se exponen en este artículo son parte de un trabajo de investigación más amplio, cuyo propósito es estudiar, mediante el protocolo DDK del MSP, el rendimiento articulatorio en personas con disartria. No obstante, para realizar dicha investigación, se hacía necesario primero contar con valores de referencia de sujetos normales. Ello permitiría tener parámetros de comparación como referencia para los resultados de las personas con patología.

De acuerdo con lo anterior, el propósito de este artículo, es exponer los resultados de un estudio normativo del rendimiento articulatorio de individuos chilenos normales, realizado mediante los cinco parámetros que entrega el protocolo DDK del programa MSP.

La intención es proporcionar a la comunidad fonoaudiológica valores de referencia de una muestra de hablantes del país para la aplicación de este instrumento.

\section{Método}

Para realizar la normalización, los criterios muestrales se basaron en repetir el procedimiento de normalización del instrumento efectuado en su país de origen (Deliyski y Delassus Gress, 1997). De acuerdo con ello, se replicó el procedimiento en la población chilena.

A este tipo de procedimiento se le denomina muestra probabilística espejo o Replica Design (ASTM, 2007) y debe consistir en colectar una muestra de la misma cantidad de sujetos del estudio con el cual se hará la comparación (en este caso particular, 39 personas) y que cumplan con criterios y características similares a los de la muestra original, tales como (en este caso): edad entre 18 y 61 años, de ambos sexos y que no estén diagnosticados con patología del habla ni otra afección neurológica. Este procedimiento permite, por un lado, realizar una comparación de resultados entre ambos estudios y, por otro, establecer valores de referencia sin tener que recurrir a muestras tan grandes.

Para la toma de los datos se utilizó un micrófono que se ubicó a una distancia estándar de entre 12 y $15 \mathrm{~cm}$ de los labios del informante y se orientó hacia la boca en un ángulo de aproximadamente $30^{\circ}$ a $45^{\circ}$. La señal de voz fue ingresada directamente al computador usando un micrófono dinámico Shure modelo SM- 58, conectado a una tarjeta de audio externa USB modelo FastTrack de M-Audio, para luego ser analizada por el software Motor Speech Profile. Este procedimiento se efectuó en un lugar apropiado y lejos de ruidos ambientales que pudieran interferir en la toma de muestras sonoras. Para obtener valores de rendimiento diadococinético, se pidió al informante que emitiera una secuencia consonante vocal ("pa") y que la repitiera de manera rápida y consistente durante la recopilación de datos.

Se determinó una muestra probabilística de tipo aleatoria, es decir, todos los elementos de la población tienen la misma probabilidad de ser escogidos (evitando sesgos). Así, se pretende obtener una muestra representativa de la población en estudio.

Los participantes se distribuyeron en un grupo de 20 mujeres y otro de 19 hombres, puesto que la 
pirámide poblacional del país no hay mucha diferencia en la distribución de adultos entre ambos géneros. Siguiendo los parámetros de la normalización modelo, hombres y mujeres se distribuyeron como se observa en la Tabla 1.

Tabla 1

Distribución de edades según género

\begin{tabular}{lll}
\hline Hombres & $27-61$ años & $\overline{\mathrm{X}}=40.5$ años \\
Mujeres & $18-60$ años & $\overline{\mathrm{X}}=34.4$ años \\
\hline
\end{tabular}

A cada participante, se le aplicó el protocolo del programa MSP para la evaluación del rendimiento articulatorio del habla. Se tomaron tres muestras del habla a los 39 sujetos y se utilizó la segunda muestra para realizar el análisis, considerando que en la primera el participante practica el sonido consonante vocal, y en la tercera muestra se añade el factor de cansancio. Los datos fueron ingresados al MSP, el cual entregó los índices DDK para cada sujeto evaluado. Luego se hizo el análisis estadístico de los datos y se obtuvo la media del índice DDK calibrado de sujetos normales.

Finalmente, con el propósito de conocer si los resultados del estudio realizado en Chile difieren o son similares a los del estudio efectuado en EE.UU., se efectuó una comparación con la prueba $t$ de Student.

\section{Resultados}

Se presentan los resultados obtenidos al analizar el habla de 39 participantes con habla normal en el primer protocolo de evaluación del software Motor Speech Profile. Los resultados para cada indicador y su comparación con el estudio norteamericano se detallan a continuación.

\section{Índice de duración promedio de la sílaba (DDKavp)}

Como se puede observar en la Tabla 2, la duración promedio de la sílaba en el total de individuos de este estudio es de 263 ms. Este valor es considerablemente más alto que el valor de referencia que viene en el programa, aunque es necesario consignar también que al observar el valor de la desviación estándar se puede ver que existe mayor dispersión en el comportamiento de los sujetos chilenos. En la clasificación por sexo, los varones promedian 228 ms, en oposición a las mujeres que promedian $297 \mathrm{~ms}$.

Tabla 2

Valores promedio y desviación estándar parámetro DDKavp

\begin{tabular}{lcccccc}
\hline & Todos & \multicolumn{3}{c}{ Mujeres } & \multicolumn{3}{c}{ Hombres } \\
\hline & $\overline{\mathrm{X}}$ & $\mathrm{S}$ & $\overline{\mathrm{X}}$ & $\mathrm{S}$ & $\overline{\mathrm{X}}$ & $\mathrm{S}$ \\
\hline Chile & 263,36 & 66,15 & 296,70 & 68,75 & 228,26 & 41,39 \\
EE.UU. & 171,18 & 15,90 & 173,81 & 17,41 & 168,54 & 14,19 \\
\hline
\end{tabular}

Al comparar los valores obtenidos en Chile con los obtenidos en EE.UU. mediante la prueba $t$ de Student, se advirtió que las diferencias son significativas, por lo tanto se puede concluir que, en cuanto a este indicador, ambos grupos se comportan de manera diferente.

\section{Promedio de sílabas por segundo (DDKavr)}

Este parámetro indica la tasa de vocalizaciones, es decir, el número de combinaciones $\mathrm{CV}$ en un segundo. Este valor, por lo tanto, es inversamente proporcional al valor que se obtiene en el parámetro DDKavp. La tasa promedio total de vocalizaciones consonante vocal en un segundo es de $4 \mathrm{~s} / \mathrm{s}$. En mujeres es de $3,5 \mathrm{~s} / \mathrm{s}$ y en hombres de $4,6 \mathrm{~s} / \mathrm{s}$ (Tabla 3). 
Tabla 3

Valores promedio y desviación estándar parámetro DDKavr

\begin{tabular}{lcrrrrr}
\hline & Todos & \multicolumn{3}{c}{ Mujeres } & \multicolumn{3}{c}{ Hombres } \\
\hline & $\overline{\mathrm{X}}$ & $\mathrm{S}$ & $\overline{\mathrm{X}}$ & $\mathrm{S}$ & $\overline{\mathrm{X}}$ & $\mathrm{S}$ \\
\hline Chile & 4,00 & 1,01 & 3,49 & 0,85 & 4,53 & 0,91 \\
EE.UU. & 5,89 & 0,50 & 5,81 & 0,54 & 5,97 & 0,46 \\
\hline
\end{tabular}

Se puede observar que, en promedio, los informantes chilenos articularon más lento que los norteamericanos, sin embargo, la dispersión en los valores es más alta en la muestra nacional. Al aplicar la prueba $t$ de Student, se pudo constatar que las diferencias entre ambos grupos son significativas, por lo tanto, respecto de este indicador, los grupos no se comportan de la misma forma.

\section{Porcentaje de variabilidad en la producción de sílabas CV en un lapso de tiempo (DDKcvp)}

El parámetro DDKcvp mide el grado de variación (o regularidad) de la tasa de producción de sílabas en un período de tiempo, es decir, este indicador mide qué tan constante y regular es el número de producciones CV ("pa" en un segundo) del informante. Este indicador se expresa en términos porcentuales, de manera que el valor que entrega corresponde al porcentaje de irregularidad o de variabilidad en la producción de sílabas. Como se aprecia en la Tabla 4, la media porcentual total es de un $5,5 \%$ de variabilidad. En varones es de un $5,4 \%$ y en mujeres de un $5,6 \%$ de irregularidad.

Tabla 4

Valores promedio y desviación estándar parámetro DDKcvp

\begin{tabular}{rccccccc}
\hline & Todos & \multicolumn{3}{c}{ Mujeres } & \multicolumn{3}{c}{ Hombres } \\
\hline & $\overline{\mathrm{X}}$ & $\mathrm{S}$ & $\overline{\mathrm{x}}$ & $\mathrm{S}$ & $\overline{\mathrm{x}}$ & $\mathrm{S}$ \\
\hline Chile & 5,48 & 1,66 & 5,56 & 1,52 & 5,39 & 1,83 \\
EE.UU. & 6,01 & 1,12 & 6,49 & 1,02 & 5,52 & 1,03 \\
\hline Al & comparar la & muestra & de & Chile & con la
\end{tabular}

norteamericana mediante la prueba $t$ de Student, se observó que en los valores de este indicador no se da una diferencia estadísticamente significativa, por lo tanto, se puede afirmar que en este parámetro ambos grupos tienen un comportamiento similar.

\section{Porcentaje de variabilidad en la producción de sílabas CV, considerando ciclo a ciclo (DDKjit)}

El parámetro DDKjit mide el grado de variación (o regularidad) de la tasa de producción de sílabas haciendo una comparación ciclo a ciclo, es decir, este indicador al igual que el DDKcvp, mide qué tan constante y regular es el número de producciones CV del informante, pero en lugar de hacerlo dentro de un período de tiempo, lo hace comparando sílaba a sílaba. Este indicador se expresa en términos porcentuales, de manera que el valor que entrega corresponde al porcentaje de irregularidad o de variabilidad en la producción de sílabas. En la Tabla 5 se aprecia que la media porcentual de variabilidad del total de informantes es de un 1,8\%. En mujeres, la variación promedio es de prácticamente un $2 \%$, versus los hombres que presentan un $1,6 \%$ de irregularidad.

Tabla 5

Valores promedio y desviación estándar parámetro DDKjit

\begin{tabular}{lrrrrrr}
\hline & Todos & \multicolumn{3}{c}{ Mujeres } & \multicolumn{3}{c}{ Hombres } \\
\hline & $\overline{\mathrm{X}}$ & $\mathrm{S}$ & $\overline{\mathrm{x}}$ & $\mathrm{S}$ & $\overline{\mathrm{x}}$ & $\mathrm{S}$ \\
\hline Chile & 1,78 & 0,66 & 1,96 & 0,62 & 1,59 & 0,67 \\
EE.UU. & 1,25 & 0,30 & 1,35 & 0,33 & 1,16 & 0,25 \\
\hline
\end{tabular}

Se puede ver en la Tabla 5 que los valores de los sujetos chilenos son más altos en general, aunque todos dentro del rango entre el $1 \%$ y $2 \%$. La dispersión de los datos, eso sí, también es más alta 
en la muestra nacional. Al comparar ambas muestras mediante la prueba $t$ de Student, los resultados indican que las diferencias entre los grupos son significativas.

\section{Coeficiente de variación de la intensidad máxima (DDKcvi)}

Este indicador mide el grado de variación de la intensidad en la cima de amplitud de cada vocalización CV. Se considera, entonces, como un correlato de la capacidad del hablante para mantener una amplitud constante, es decir, evalúa si el sujeto es capaz de mantener cierta regularidad en la fuerza espiratoria durante la ejecución de la tarea. Este indicador se expresa en términos porcentuales, de manera que el valor que entrega corresponde al porcentaje de irregularidad o de variabilidad de las cimas de amplitud de la producción de la fase vocálica de la tarea. Como se observa en la Tabla 6, el valor promedio de variabilidad en mujeres es de un $2 \%$ y en hombres es de un $1,7 \%$, mientras que la media en la totalidad de los individuos es de un $1,8 \%$.

Tabla 6

Valores promedio y desviación estándar parámetro DDKcvi

\begin{tabular}{lcrrrrr}
\hline & Todos & \multicolumn{3}{c}{ Mujeres } & \multicolumn{3}{c}{ Hombres } \\
\hline & $\overline{\mathrm{X}}$ & $\mathrm{S}$ & $\overline{\mathrm{X}}$ & $\mathrm{S}$ & $\overline{\mathrm{x}}$ & $\mathrm{S}$ \\
\hline Chile & 1,84 & 0,75 & 2,02 & 0,68 & 1,66 & 0,79 \\
EE.UU. & 1,91 & 0,64 & 1,97 & 0,59 & 1,85 & 0,70 \\
\hline
\end{tabular}

En general, se puede constatar que todos los valores son cercanos al $2 \%$ de variabilidad en este parámetro y que el grado de dispersión de los datos en ambos grupos es bastante similar. Al efectuar la comparación con la prueba $t$ de Student, se observó que las diferencias no son significativas, por lo tanto, se puede concluir que en este indicador los comportamientos de ambas muestras son similares.

\section{Conclusión}

En síntesis, el coeficiente de variación del período DDK (DDKcvp) y el coeficiente de variación de la intensidad máxima (DDKcvi) son los parámetros más estables, en el sentido de que su comportamiento es similar tanto en la normalización original del software como en la normalización realizada en este estudio.

Se puede afirmar que, en sujetos normales, la duración promedio de la sílaba (DDKavp) debería oscilar entre los 200 y 300 ms, el promedio de producción de sílabas por segundo (DDKavr) debería variar entre 4 y 5, la variabilidad de la producción de sílabas medida en intervalos temporales no debería ser más alta que un $5 \%$ a $6 \%$ y el porcentaje de variabilidad, tanto de la producción de sílabas comparando sílaba a sílaba (DDKjit) como de la intensidad máxima (DDKcvi), no debería superar el $2 \%$.

Los resultados generales del estudio demuestran que el instrumento es sensible a variaciones mínimas y detalladas del habla que el oído humano no puede identificar con facilidad. La normalización permite fomentar el uso y aplicación del instrumento con el objeto de respaldar el diagnóstico y evaluación de patologías del habla y también de visualizar mejorías y progresos de los individuos en sus tratamientos y terapias.

El alcance del instrumento normalizado tiene múltiples aristas, y sin duda significará una gran ayuda al profesional terapeuta. 


\section{Referencias}

ASTM. (2007). E122-07. Standard practice for calculating sample size to estimate, with specified precision, the average for a characteristic of a lot or process. Recuperado de

http://www.astm.org/DATABASE.CART/HISTORICAL/E122$\underline{07 . h t m}$

Brisso, V. (2007). Tasa de diadococinesia en sujetos con fisura (Tesis de pregrado inédita). Universidad de Talca, Talca, Chile.

Deliyski, D. \& Delassus Gress, C. (noviembre, 1997). Characteristics of motor speech performance: Normative data. ASHA'97, Boston, Massachusetts. Recuperado de http://www.sph.sc.edu/Documents/1997ASHAhandout.pdf

Harel, B., Cannizzarro, M., Cohen, H., Reilly, N., \& Snyder, P. (2004). Acoustic characteristics of Parkinsonian speech: A potential biomarker of early disease progression and treatment. Journal of Neurolinguistics, 17(6), 439-453.

Jeen, J., Kanaka, G., Sunila, J., \& Bellur, R. (2014). Normative for Motor Speech Profile in Kannada- speaking adults. Journal of Voice, 28(1), 7-13.

Louzada, T., Beraldinelle, R., Berretin, F., \& Brasolotto, A. (2011). Oral and vocal fold diadochokinesis in dysphonic. Journal of Applied Oral Science, 19(6), 567-572.
Lundy, D., Roy, S., Xue, J., Casiano, J., \& Jassir, D. (2004). Spastic/spasmodic vs. tremulous vocal quality: Motor Speech Profile analysis. Journal of Voice, 18(1), 146-152.

Meurer, E. M., Wender, M. C. O., Corleta, H. E., \& E. Capp, E. (2004). Female suprasegmental speech parameters in reproductive age and postmenopause. Journal Maturitas, 48(1), 71-77.

Padovani, M., Gielow, I., \& Behlau, M. (2008). Phonarticulatory diadochokinesis in young and elderly individuals. Arquivos de Neuro-Psiquiatria, 67(1), 58-61.

Queiroz, M. (2002). Fundamentos de Fonoaudiología. Sao Paulo: Editorial Panamericana.

Toledo, L., Bahamonde, C., González, J., Martínez, M., Muñoz, M. M., y Muñoz, D. (2011). Parámetros del habla en adultos normales chilenos. Revista Chilena de Fonoaudiología, 10, 33-43.

Vergara, C. (2008). Tasa de diadococinesia en niños típicos hablantes de español de entre 10 años y 13 años 11 meses de edad: datos normativos (Tesis de pregrado). Universidad de Talca, Talca, Chile.

Wang, Y., Kent, R., Duffy, J., \& Thomas, J. (2008). Analysis of diadochokinesis in ataxic dysarthria using the Motor Speech Profile program. Folia Phoniatrica et Logopaedica, 61, 1-11.

Williams, P. \& Stackhouse, J. (2000). Rate, accuracy and consistency: Diadokokinetic performance of young, normally developing children. Journal of Clinical Linguistics and phonetics, 14, 267-29. 